THE KURUME MEDICAL JOURNAL Vol. 3, No. 3, 1956

\title{
A NEW FREEZE-DRYING APPARATUS FOR ELECTRON MICROSCOPICAL SAMPLING*
}

\author{
K. TOKUYASU \\ Department of Anatomy, School of Medicine, Kurume University, \\ Kurume-shi, Japan
}

\section{INTRODUCTION}

It will be the general recognition that the freeze-drying tecnique is a very powerful method for studying the morphological or chemical compositions of tissues or microbes if the technique can be applied properly to each case with a particular purpose (e. g. (1)). For the morphological examination, particularly with the electron microscope, it is very necessary to minimize the size of ice grain to avoid artifacts during the freezing process. The only way to achieve this purpose at present is to cool the sample as rapidly as possible to a very low temperature by some method or other and it is desirable to carry out the sublimation of ice without a much raise of temperature since the raise may cause the re-crystalization of ice crystals and accordingly some kind of artifact (2). The water vapor pressure of ice is so low at a temperature such as -50 to $-100^{\circ} \mathrm{C}$ that a high vacuum such as $10^{-4}$ to $10^{-6} \mathrm{mmHg}$ may be necessary for the sublimation of ice with an ordinary closed-chamber system. Since the evacuation of water vapor from the sample is to condense the vapor at the trap in the path of evacuation, a higher vacuum and a closer distance between the sample and the trap may accelerate more the diffusion rate of vapor from the sample to the trap. If the complexity of a high vacuum system may be avoided for a technical simplicity or an economical consideration, a lowered rate of evacuation may result because of the sacrifice of the vacuum degree but the shortening of the distance between the sample and the trap may compensate at

*This study was carried out mainly at the Department of Bacteriology, School of Medicine, Kyushu University and the Department of Infectious Diseases. School of Medicine, University of California at Los Angeles and was presented at the meeting of Kyushu Chapter of Bacteriological Society of Japan held in Miyazaki, October 1956. 
least a part of the lowered rate.

In fact, it was reported that this method of evacuation worked well (3). The construction of a special shape of the trap, however, was necessary for this method and the technical control of the system did not seem so easy since the distance between the sample and the trap had to be adjusted properly and the admittance of wet air onto the trap surface seemed to have to be avoided carefully before and after drying of the sample if the trap was to be used repeatedly.

A greater gradient of vapor concentration at the surface of sample gives a higher rate of sublimation of ice and there may be two ways for obtaining a greater gradient. One is to maintain the outside of the sample at a higher vacuum so that the sublimated vapor may diffuse more easily toward the trap without being interfered by the existence of other kinds of gases. The other is to wash the surface of sample with the flow of some dry gas, and a greater rate of flow may provide a greater gradient, speaking at the practical sense. The first one is the type being commonly used for the purpose of freeze-drying and the second is practised for general drying purposes of various goods. The preservation of morphological compositions of sample may not be maintained if the sample is exposed to a strong flow of gas under the atmospheric pressure but if the pressure is dropped to a properly low value, the usability of the second method seems to remain to be checked.

As a matter of fact, when a frozen biological sample was kept in a glass cylinder and a moderate flow of dry nitrogen or carbon dioxide was allowed to pass over the sample surface, missing of parts of sample or some kinds of artifacts were observed after drying. Contrary to this, a successful experiment using the vacuum of $15 \mathrm{mmHg}$ was reported by W. A. Jensen (4), during the author's trial with the same kind of ideas. He reported that the necessary length of time for the drying could be reduced to about one twentieth of the one needed at the ordinary instrument.

Since even such an order of vacuum as $15 \mathrm{mmHg}$ seemed inadequate for the preservation of fragile structures necessary for the observation by the electron microscope, such a higher vacuum as 0.5 to $1 \mathrm{mmHg}$ was emplyed at the present study and the various points were improved to increase the efficiency of evacuation and the convenience of the practical operation. The present paper is to report the structure of the system 
and several results obtained by means of the system.

\section{INSTRUMENT}

For a rough checking, it is assumed that the sublimation of ice is carried on at $-50^{\circ} \mathrm{C}$. Since the vapor pressure of ice at this temperature is about $3 \times 10^{-2} \mathrm{mmHg}$, the partial pressure of water vapor in the dry gas to be used must be lower than this value.

Fig. 1

Models for making dry-air flow

(A)
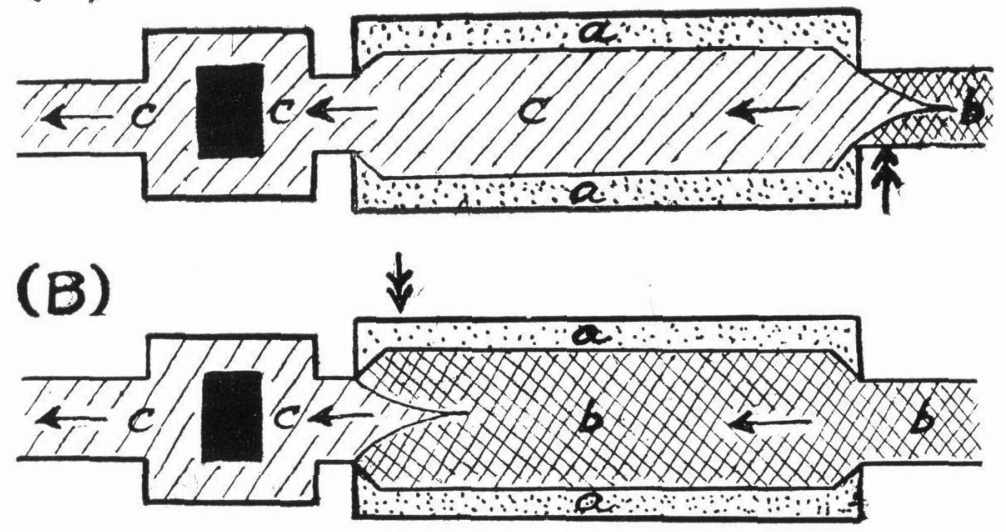

a; cooling bath of trap, b; atmospheric pessure, c; vacuum of 1 $\mathrm{mmHg}$, arrows; dirction of flow, and double-arrows; positions of capillaries.

Fig. $1 \mathrm{~A}$ is the scheme of the model in which, through a capillary, air gets in the water trap kept at the temperature of $-70^{\circ} \mathrm{C}$ and at the vacuum of $1 \mathrm{mmHg}$, and the cooled dry air of the lowered pressure washes the sample. Fig. 1B is the one in which air gets directly in the trap and air of the atmospheric pressure thus cooled and dried is sucked in the vacuum part of $1 \mathrm{mmHg}$ to wash the sample.

The air cooled down to $-70^{\circ} \mathrm{C}$ contains the partial pressure of water vapor of about $2 \times 10^{-3} \mathrm{mmHg}$ and this value seems to be sufficiently low being compared with the value of $3 \times 10^{-2} \mathrm{mmHg}$ since the difference between $3 \times 10^{-2}$ and $2 \times 10^{-3}$ is about nine tenth of the one between $3 \times 10^{-2}$ and zero corresponding to the perfectly dry condition. 
At the model of Fig. 1A, the partial pressure of water vapor will be such a value as shown above and accordingly, this model may seem to be practical.

As a matter of fact, this model was practised at the preliminary experiment and was proved to be usable. Technically speaking, however, it did not seem so easy to assure that the whole volume of air with a fairly high speed have reached equally to $-70^{\circ} \mathrm{C}$.

At Fig. 1B, the partial pressure of water vapor will drop when the atmosheric pressure of air in the trap is dropped to $1 \mathrm{mmHg}$ and the ratio of the dropping will be about the same both for the air and its constituent gases. This means that the air with the partial pressure of water vapor of $2 \times 10^{-3} \times 760 \mathrm{mmHg}$ may have the partial pressure of $2 \times 10^{-3} \mathrm{mmHg}$ after passing through the capillary. In other words, even when the partial pressure of water vapor in the trap may be such a high value as 1.5 $\mathrm{mmHg}$, the partial pressure at the part of sample will be such an order of $2 \times 10^{-3}$ mmHg. Considering as above, it may be clear that the model of Fig. $1 \mathrm{~B}$ is much safer than the one of Fig. 1A to obtain dry air for the purpose.

The temperature of the mixture of dry ice ( solid $\mathrm{CO}_{2}$ ) and ethylalcohol is $-72^{\circ} \mathrm{C}$ and the mixture may be used for the bath of the water trap. A copper rod is fitted to a hole opened in the rubber stopper for the vacuum and the flat top of the rod serves as the specimen holding part. The extension of the rod at the other side is immersed in the mixture mentioned above and the temperature of the rod top is controlled by adjusting the relative distance between the top and the meniscus of the mixture. To know the temperature of the top, a thermo-couple or a thermistor thermometer may be attached there. Or, for practical operations under an indifferent condition, a preliminary experiment may be carried out to plot the temperature against the distance between the top and the meniscus of the cooling mixture, and from the curve plotted the temperature may be approximately estimated. The mutual relationship of arrangement of parts is shown in Fig. 2.

The rotary vacuum pump which was employed at the present study had the evacuation rate of $2001 / \mathrm{min}$. and the size of glass capillary for the air leak was so adjusted that the vacuum of the sample part was kept at the vacuum between 0.5 and $1 \mathrm{mmHg}$. The degree of vacuum was read from the discharging pattern of a Geisler tube and commercial thermoses were used as the containers of cooling mixture. The 
Fig. 2

Arrangement of parts

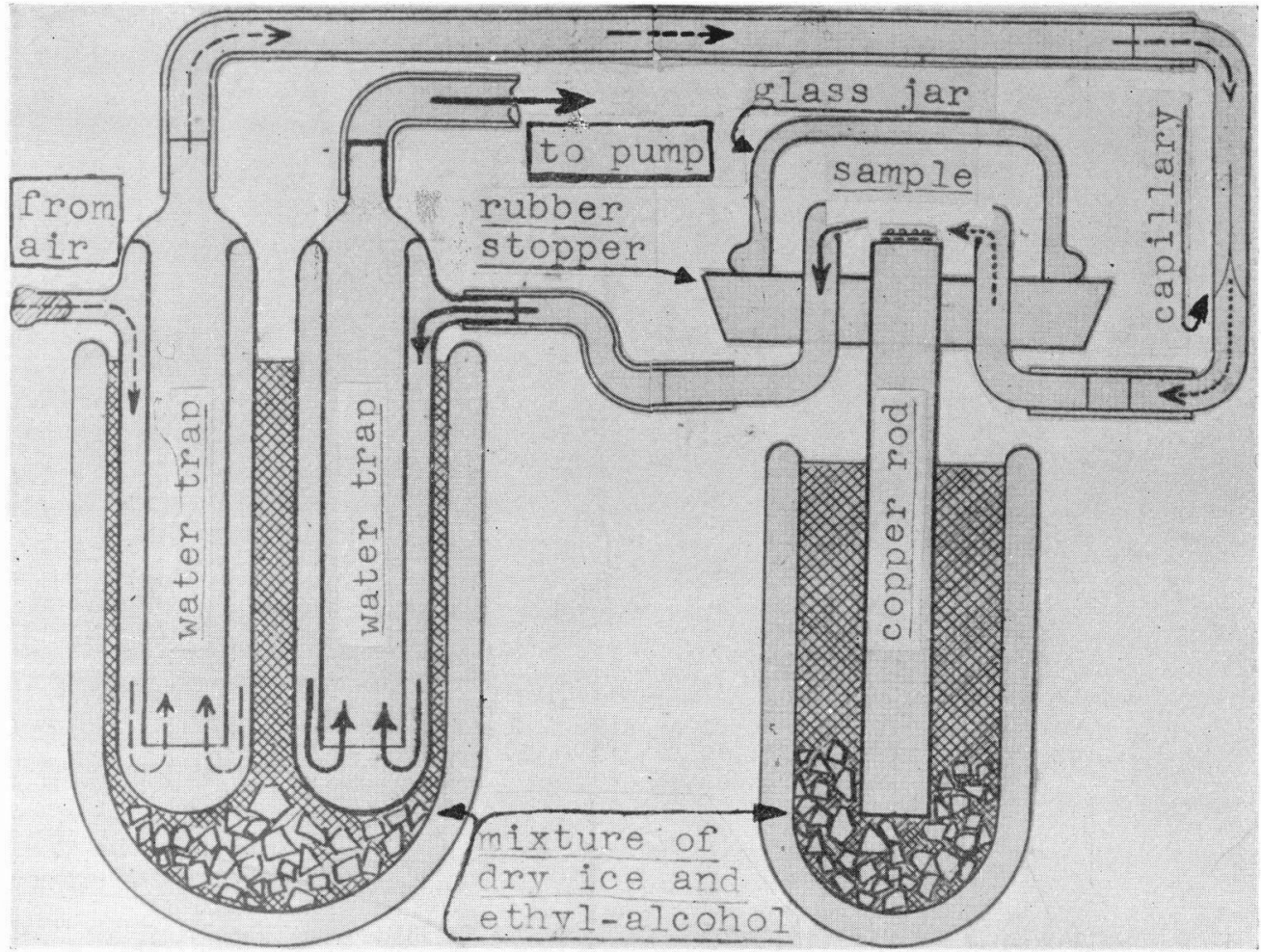

Each part is indicated in chart.

water vapor carried by the flow of dry air was captured at another trap before the evacuation pump.

\section{EXPERIMENT AND RESULTS}

To examine the capability of the instrument, several drops of about $2 \mathrm{~mm}$ diameter of water were frozen on the rod top being kept at about $-50^{\circ} \mathrm{C}$ and the sublimation of these ice drops were carried out. It was observed that they were sublimated within 1 to 2 hours. Repeating experiments, the strict washing of the sample surface only did not appear to give a better result than a milder ventilation of the sample-chamber as a whole.

For checking the possible artifacts by air flow over the sample, a kind of bacte- 
riophage* and Leptospira hebdmadis $A^{* *}$ were chosen to be examined. At first, these samples were fixed by $1 \%$ buffered solution of $\mathrm{OsO}_{4}$ to help the preservation of morphological structures during the freezing process and droplets of distilled water suspension of samples thus fixed were dropped on meshes covered with the collodion film. Then, the meshes were put on a thick copper disk which had been preliminarily cooled on the top of a block of dry ice. The droplets of samples froze immediately and the copper block was transfered to the top of copper rod described before. The evacuation was carried out about 1 to 3 hours and the cooling bath of copper rod was removed from the position. When the rod seemed to have reached to the room temperature, the bath of hot water was given to the rod to assure that the sample was warmer than the room air and that air moisture would not condense on the sample. The sample chamber was then separated from other parts of the apparatus and room air was introduced into the chamber to remove meshes from the top of rod. Meshes were immediately transfered into the shadowing apparatus and a moderate amount of shadowing by $\mathrm{Cr}$ was given to samples. Then, these samples were examined by the electron microscope (Hitachi $\mathrm{HU}-9$ and -6 ).

Figs. 3-5 are several examples of results thus obtained. At Fig. 3, it is clear that the hexagonal shape of the bacteriophage head was kept well. Fig. 4 is to show that the three-dimensional helical structure of leptospira was well preserved, the shadow of each loop of helix being thrown on the ground (being indicated by arrows). It is shown in Fig. 5 that the standing position of the possibly fragile structure of leptospira could be remained in air.

These evidences mentioned above seemed enough to suggest that artifacts could be caused but very little, by the action of flow upon the sample.

Fig. 3

Note the hexagonal shape of bacteriophage head, $100,000 \times$

Fig. 4

Each loop of helix of leptospira casts its shadow on ground, $30,000 \times$

*The sample was given by Dr. M. I. Sellers, University of California, during the author's stay at the school.

**The sample was given by Dr. R. Mori, Kyushu University. 


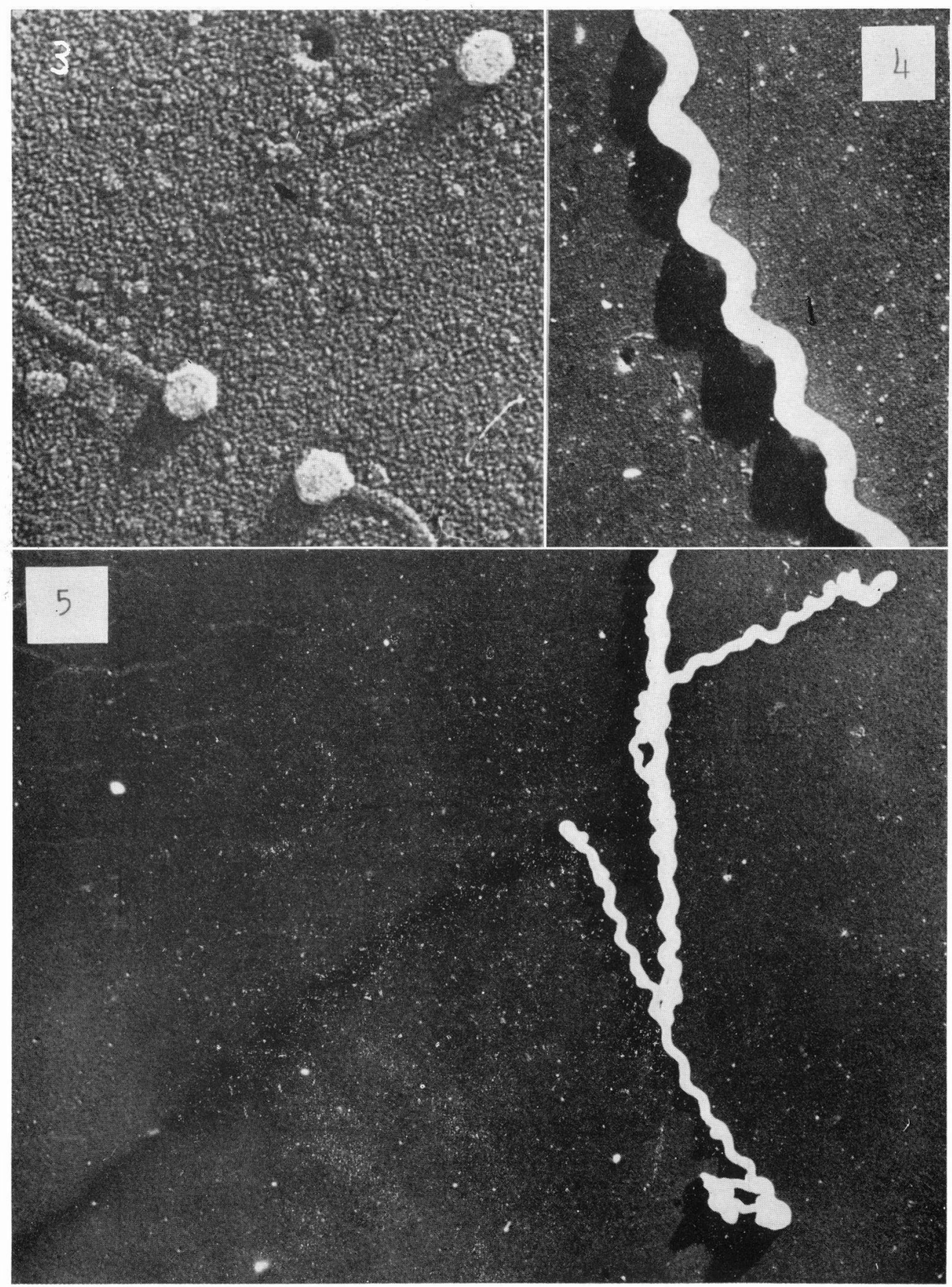


Fig. 5

The standing bacterial body of leptospira at the upper right corner casts its long shadow toward the lower left corner, $12,000 \times$

\section{DISCUSSIONS}

As the results show, the instrument seems to be very efficient and practical at least for applications of small scale, although it is not yet tested for freeze-drying of tissues. At the present study, the spraying method founded by R. C. Williams for very quick freezing of tiny objects such as viruses (1) was not employed but the hexagonal shape of bacteriophage head was well preserved as shown in Fig. 3. This seems to suggest that the fixation helps the preservation of morphological structure, protecting the deformation by the surface tension of water possibly occuring under a much slower freezing rate than the one at the spraying method.

Since this method of freeze-drying has only a little of experience comparing with the ordinary closed-chamber apparatus, various aspects upon the physical characteristics or so on seem to be better discussed after getting a sufficient amount of data, and a further discussion is omitted here.

\section{SUMMARY}

1. A freeze-drying apparatus which utilized the drying effect of dry gas flow rather than the vacuum itself was designed and tested.

2. The drying efficiency of the instrument seemed to be much better than the one of the ordinary closed-chamber system.

3. The possible artifact by the gas flow was checked using such fragile samples as bacteriophage or leptospira and was proved to be at least negligibly small.

\section{ACKNOWLEDGEMENT}

The author wants to give his deep appreciation to Prof. T. Toda and Dr. R. Mori of Kyushu University and Prof. C. M. Carpenter and Dr. M. I. Sellers of University of California at Los Angeles. 


\section{REFERENCES}

1. Williams, R. C.: A method of freeze-drying for electron microscopy. Exp. Cell Res., 4, 188, 1953.

2. Meryman, H. T.: Mechanics of freezing in living cells and tissues. Science, 124,515 , 1956.

3. FreEd, J. J.: Freeze-drying technics in cytology and cytochemistry. Lab. Invest., 4, $106,1955$.

4. Jensen, W. A.: A new approach to freeze-drying of tissue. Exp. Cell Res., 7, 572, 1955. 